\title{
Protection Strategy against IEMI for Wireless Communication Infrastructures
}

\author{
Stefan van de Beek*, Mirjana Stojilović†, Nicolas Mora ${ }^{\ddagger}$, \\ Marcos Rubinstein ${ }^{\dagger}$, Farhad Rachidi-Haeri ${ }^{\ddagger}$, and Frank Leferink ${ }^{*}, \S$ \\ *University of Twente, Enschede, Netherlands, g.s.vandebeek@utwente.nl \\ †University of Applied Sciences and Arts Western Switzerland, Yverdon-les-Bains, Switzerland, \\ ${ }^{\ddagger}$ Swiss Federal Institute of Technology (EPFL), Lausanne, Switzerland, \\ $\S$ Thales Nederland B.V., Hengelo, Netherlands
}

\begin{abstract}
In this paper, we present a methodology for estimating the protection levels of critical equipment against IEMI. We apply the method to a wireless communication infrastructure, but it can be applied to any critical infrastructure. The required protection levels that need to be implemented are determined by analyzing the susceptibility of the victim, and the threat level associated with a specific IEMI scenario. Based on the required protection levels, possible protection strategies are identified and the associated costs are evaluated. The total cost includes the monetary cost and the costs related to loss of performance. The method we present can be used for a quantitative comparison between different protection techniques in terms of monetary cost. The method is applied to develop a strategy for protecting a wireless receiver from damage due to in-band interference using an RF limiter. We also analyze the loss in performance due to the installation of a limiter. It is shown that the noise figure degradation is linearly dependent on the insertion loss of the limiter.
\end{abstract}

\section{INTRODUCTION}

The use of wireless communications has seen a steep increase over the recent decades. Large, distributed infrastructures provide wireless coverage over most parts of the world. The most widely used wireless infrastructure is the cellular radio network. This system finds its applications in many standards for radio communication, such as Terrestrial Trunked Radio (TETRA), Global System for Mobile Communications (GSM), Universal Mobile Telecommunications System (UMTS), and Long Term Evolution (LTE). A variety of mission-critical communication applications, for example the public safety communications, are based upon the TETRA standard and, as such, it is considered to be a critical infrastructure.

A possible threat against critical infrastructures is intentional electromagnetic interference (IEMI) [1]. In [2], the wireless communication infrastructure was analyzed to find the most critical subsystems in case of an electromagnetic attack. The wireless link and the wireless receivers were recognized as the most vulnerable subsystems. The base station transceiver is particularly critical, since its failure can bring down a complete cell. Besides that, a base station is often an easy target since it is usually located at a fixed position on a tall mast, which provides a line-of-sight link to the attacker [3]. It is difficult to protect the receivers and the wireless link against IEMI for various reasons. First of all, due to the open access nature of 978-1-5090-1416-3/16/\$31.00 (c) 2016 IEEE the shared medium, the wireless link can be easily jammed by interference within the frequency band of operation. Secondly, the electronics of the receiver are difficult to shield against IEMI since the antennas are intended as a point of entry for electromagnetic energy. The coupling of IEMI via the antenna is defined as front door coupling [4].

Several studies have been carried out to investigate the vulnerability of wireless communication systems against IEMI. In [5], the vulnerability of the GSM-R standard (global system for mobile communications (GSM) standard for railway usage) was studied, and in [6], the vulnerability of a TETRA base station was investigated. Both papers focused on the expected threat levels due to IEMI, and on the susceptibility levels of the equipment. In this paper, we extend the work in [5] by providing a systematic analysis to determine the required protection levels. This overall analysis methodology is applicable to any critical infrastructure, i.e., it is not restrained to the wireless communication infrastructure. Based on the required protection levels, we identify applicable protection techniques for wireless communication. Finally, we investigate the associated costs, both monetary and due to loss of performance, associated with the implementation of the protection techniques. The method to assess the monetary costs can be used for a quantitative comparison of different protection techniques that are suitable for protection of the infrastructure. We are mainly interested in presenting a method that can be used to compare different techniques and not in the exact assessment of the costs.

This paper is organized as follows. The expected power at the input of a base station receiver and its susceptibility levels are discussed in Section II. The procedure for estimating the protection levels is presented in Section III. The application of the methodology to the case of a base station is shown in Section IV. The possible protection schemes and their selection based on costs are discussed in Sections V and VI, respectively. Finally, conclusions and perspectives are given in Section VII.

\section{Vulnerability Analysis of a Base Station RECEIVER}

In this section, we analyze the vulnerability of a typical base station receiver against IEMI, based on information available in the literature and on previous research [6]-[9]. We start by analyzing the power transfer from the IEMI source to the susceptible electronics in the receiver. Then, we discuss the selectivity of receivers and interference mechanisms. 


\section{A. Transfer Function}

Since it is directly connected to the antenna, the front end of the receiver is most susceptible to IEMI. Assuming far field conditions, the received signal power of the antenna equals [10]

$$
P_{\mathrm{rx}}=\frac{E^{2}}{Z_{0}} \frac{\lambda^{2}}{4 \pi} G(\theta, \phi)\left(1-|\Gamma|^{2}\right) e_{\mathrm{p}},
$$

where $E$ is the RMS value of the electric field at the antenna, $Z_{0}$ is the wave impedance, $\lambda$ is the wavelength, $G(\theta, \phi)$ is the gain of the antenna as a function of the polar and azimuthal angle, $\Gamma$ is the antenna reflection coefficient, and $e_{\mathrm{p}}$ is the polarization mismatch.

From (1), we can make two important observations. Firstly, the received power is space-dependent and related to the antenna pattern. The maximum amount of energy is received if the direction of the interferer is along the boresight of the receiving antenna. This is one of the reasons why front door interference can be relatively easily achieved at a large distance. The gain of the receiving system can be used by the adversary to effectively couple IEMI into the system. Secondly, the received power is strongly frequency dependent due to the antenna reflection coefficient. An antenna is often designed such that the coefficient is below $-10 \mathrm{~dB}$ for the desired frequencies, i.e. in-band frequencies. For out-of-band (OoB) frequencies the reflection coefficient can be higher, resulting in less received power. However, antennas can be very broadband or can have more resonating frequencies with a low reflection coefficient.

After the signal is received by the antenna, it is further processed by the front end of the receiver. The front end is almost always directly located at the antenna so that there is no need for a long cable. This improves the noise performance of the receiver [11]. Typically, the RF signal is first filtered by a front door filter which selects an entire band (the bandwidth typically ranges from a few $\mathrm{MHz}$ to $20 \mathrm{MHz}$ ). Next, the signal is fed to a low noise amplifier (LNA). Then, the mixer converts the amplified signal to a lower IF frequency where it is easier to implement a sharp analog filter to suppress out-of-channel interference. Finally, the signal can be amplified again and converted to the digital domain for further processing.

\section{B. Selectivity}

The selectivity of a receiver can be defined as the ability to correctly detect and decode a desired signal in the presence of unwanted signals close in frequency. The selectivity is achieved by implementing high quality filters that attenuates all other frequencies except those in the desired frequency channel. The channel-selection filtering is typically done at lower IF frequencies, since the required value of $Q$ is then lower. Clearly, all stages in the receiver preceding the channel selection filter should be sufficiently linear, to avoid non-linear effects, such as compression and intermodulation, resulting from unwanted spectral components.

\section{Interference Mechanisms}

In [6], it is showed that there are three interference mechanisms: damage, saturation, and jamming.
1) Damage: Firstly, high-power interference can damage the LNA [5], leading to a permanent DoS. The typical destruction level of LNAs [7] is $34 \mathrm{dBm}$, in the case of a narrowband interference with a pulse width exceeding $1 \mu \mathrm{s}$. OoB interference can be attenuated by a front filter and the damage can be avoided. However, in-band interference cannot be filtered out.

2) Saturation: Secondly, a high-power interferer (below damage levels) can saturate the receiver and as a result desensitize the receiver for the desired signal [8]. A highly selective receiver is not more robust against this interference mechanism: the degradation happens at the RF stage while the selectivity is at the IF stages. A high dynamic range of a receiver does improve the robustness against saturation. In [6], a particular base station was tested for saturation levels and it showed saturation starting from an input power at $-4 \mathrm{dBm}$.

3) Jamming: Finally, a low-power interferer, i.e. with comparable power levels as the desired signal, can mask the desired signal [9] and cause temporary denial-of-service (DoS) in the wireless communication system. If the signal-to-noise ratio is too low, the receiver will not be able to detect and decode the signal. The effectiveness of a jamming attack depends on the jamming-to-signal ratio [12]. A highly selective receiver is more robust against jamming. For an in-channel interference, protection strategies lie in spread spectrum techniques, smart antennas, and error coding schemes.

To better understand these three mechanisms, one should analyze the frequency content of the radiated IEMI. A front door coupled IEMI can be defined as in-band if the frequency is within the pass band of the front end filter or of the antenna. Otherwise, it is an OoB interference. For example, the front end filter of a TETRA base station often has a bandwidth of $5 \mathrm{MHz}$, but the bandwidth of a TETRA communication channel is only $25 \mathrm{kHz}$. This means that in-band interference can be both in-channel or out-of-channel. In Fig 1, the various frequency domains of IEMI are graphically presented. The effect of out-of-channel interference on the performance of a receiver depends on its selectivity. A wideband interference can inject its energy over the whole channel. In case of wideband interference, the total power at the input of the LNA can be approximated by integrating the power density of the interference over the bandwidth of the front end filter.

\section{Procedure For Estimating THE REQUiRED PROTECTION LEVELS}

In this section, we set up a general procedure to assess the required protection levels for a critical infrastructure.

First, one has to collect relevant information regarding the infrastructure:

1) The typical configurations of the topological and interaction sequences diagrams of the infrastructures under study;

2) The typical attenuation levels imposed by the different EM barriers that will be encountered by a travelling wave while it propagates towards the infrastructure;

3) An estimation of the radiated and conducted susceptibility levels of the critical equipment according to what has been reported in the literature and standards. 


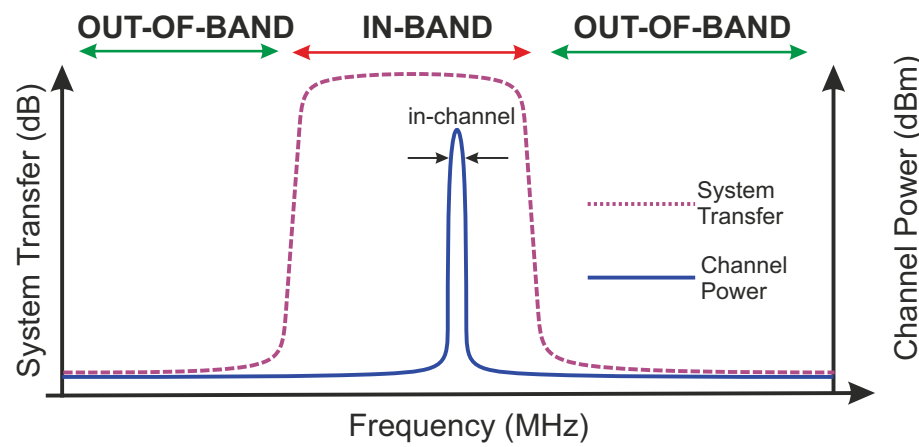

Fig. 1. Graphical representation of the various frequency domains for IEMI with respect to the system transfer (from antenna to LNA). Adopted from [6].

In the previous section, we already provided part of this information concerning a wireless infrastructure. Then, one has to implement the following steps:

(i) Create a possible scenario by choosing an IEMI source and the configuration of the EM topology; starting from the source up to the critical equipment (victim).

(ii) Obtain the far voltage, that is, the product of the peak electric field measured in the far field and the range, of the IEMI source and its fundamental frequency.

(iii) Obtain the typical transfer functions of the EM barriers found in the topological diagram.

(iv) Obtain the susceptibility threshold of the equipment.

(v) Calculate the total transfer function by adding the transfer functions of all the successive EM barriers.

(vi) Estimate the total received voltage/field at the equipment level.

(vii) Calculate the difference between the received voltage/field and the susceptibility threshold; and add the required extra protection in order to ensure compatibility.

In the first step of this procedure, the scenario is selected based upon a possible threat (realistic IEMI source) and a realistic source location. A list of potential IEMI sources can be found in [13], where the sources are assessed and characterized based on both technical and non-technical attributes. Nontechnical attributes such as availability and portability help provide an estimate of the actual risk of the analyzed scenario. Given the source characteristics and its location, the procedure will result in an estimated total received voltage/field at the equipment level. If this exceeds the susceptibility threshold, the protection needed is calculated using the difference between the received signal strength and the susceptibility level. We suggest to add a $10 \mathrm{~dB}$ safety margin to ensure compatibility. The whole procedure can be iterated for different scenarios.

\section{Required Protection LeVels For Typical Base STATION}

In this section, we calculate the required protection levels for a typical TETRA base station using the previously described procedure. The goal is to protect the wireless receiver against damage. The critical component is the LNA in the front end. We assume that its damage level is $34 \mathrm{dBm}$ (in this case we use a threshold in terms of power rather than voltage). This is, clearly, an estimate. The LNA damage level can vary for different types of LNA.

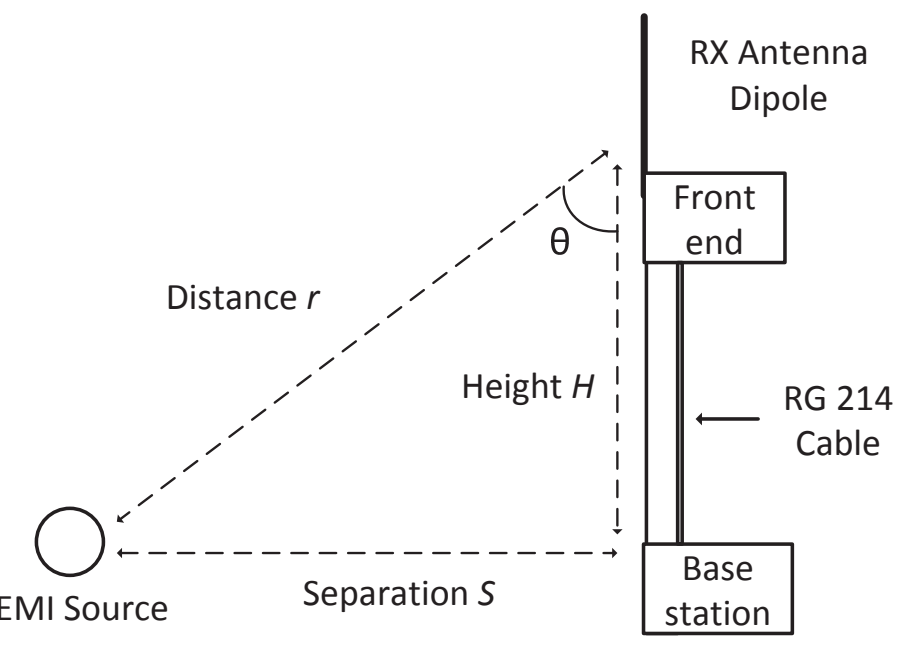

Fig. 2. EM topological diagram of the front-door illumination of the base station receiver.

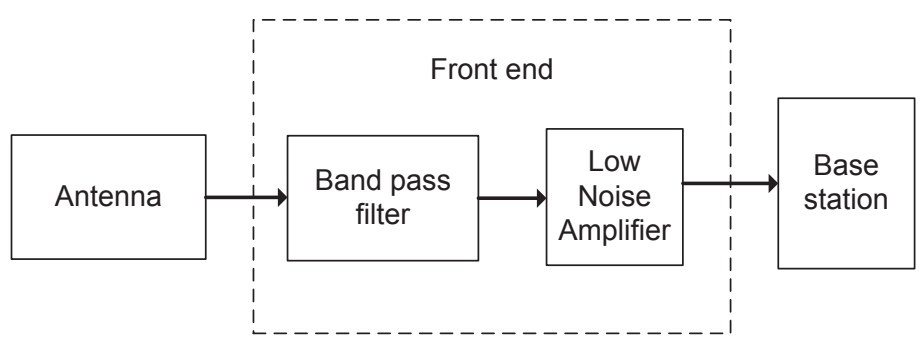

Fig. 3. Typical structure of the front end at a TETRA base station.

\section{A. Topological Decomposition of the IEMI Scenario}

The topological diagram of the proposed scenario is presented in Fig. 2 and is inspired by typical setup of a TETRA base station. A dipole antenna located on a tall mast is being used to receive the wireless communication signals. We assume it is at a height $H$ of 10 meters above ground. The antenna is directly connected to a front end, whose typical structure is shown in Fig. 3. This structure can vary depending on the manufacturer. The base station, where the actual digital processing is done, is at ground level. The front end and the base station are connected via an RG214 cable. Finally, we assume the TETRA base station operates in the 380 to 385 $\mathrm{MHz}$ frequency band.

\section{B. IEMI Source Description}

To choose the sources for this case, we have considered transportable devices that could be brought close to the station by a person or by a vehicle. We specify the generated field strengths by the far voltage, which is defined as the product of the peak electric field measured in the far field and the range (distance). In this analysis we study the effect of two different sources assumed to be at a distance $r$ of 20 meters (see Fig. 2). This implies that the separation between the source and the mast $S$ is $17.3 \mathrm{~m}$. The first source is a standard T DIEHL HPEM case [14]. It is a mesoband source and it can be tuned to operate at a frequency of $395 \mathrm{MHz}$ providing a far voltage of $225 \mathrm{kV}$. The frequency was purposely chosen as an OoB frequency. The second source is the EPFL switching oscillator connected to a monopole antenna [15]. It is a mesoband 
TABLE I. PARAMETERS OF THE CONSIDERED SOURCES

\begin{tabular}{c|c|c|c|c}
\hline $\begin{array}{c}\text { Source } \\
\text { ID }\end{array}$ & $\begin{array}{c}\text { Source } \\
\text { type }\end{array}$ & Description & $\begin{array}{c}f \\
(\mathbf{M H z})\end{array}$ & $\begin{array}{c}\text { Far voltage } \\
(\mathbf{k V})\end{array}$ \\
\hline \hline $\begin{array}{c}\text { Source } \\
1\end{array}$ & Mesoband & $\begin{array}{c}\text { A standard T Diehl HPEM } \\
\text { case. }\end{array}$ & 395 & 225 \\
\hline $\begin{array}{c}\text { Source } \\
2\end{array}$ & Mesoband & $\begin{array}{c}\text { Switching oscillator }+ \\
\text { Monopole antenna }\end{array}$ & 385 & 6.6 \\
\hline
\end{tabular}

source that is tuned at the frequency of $385 \mathrm{MHz}$, in-band, and generates a far voltage of $6.6 \mathrm{kV}$. A summary of the parameters of the considered sources is presented in Table I.

\section{Obtaining the Required Protection Level}

At a distance of $20 \mathrm{~m}$, the incident field strength of Source 1 and Source 2 will be respectively $11.25 \mathrm{kV} / \mathrm{m}$ and $0.33 \mathrm{kV} / \mathrm{m}$. The received power is calculated using (1). As a receiving antenna we use a half-wave dipole antenna which is commonly used by TETRA networks [16]. It has an $8 \%$ bandwidth from $380 \mathrm{MHz}$ up to $410 \mathrm{MHz}$ with a reflection coefficient $\Gamma$ of $0.316(-10 \mathrm{~dB})$. The gain of an omnidirectional dipole antenna is only dependent on the angle $\theta$, see Fig. 2, and is given by [10]:

$$
G(\theta)=1.64\left[\frac{\cos \left(\frac{\pi}{2} \cos \theta\right)}{\sin (\theta)}\right]^{2}
$$

The maximal gain is at $\theta=0$ and is $1.64(2.15 \mathrm{dBi})$. Furthermore, we set $Z_{0}$ at $377 \Omega$ and we assume the polarization mismatch $e_{\mathrm{p}}$ between the transmitter and the receiver to be 0.75 .

The received power is attenuated by the BPF before the LNA. A typical filter characteristic of the band pass filter [6] is presented in Fig. 4. It shows an OoB attenuation of over $80 \mathrm{~dB}$, and an in-band insertion loss of $1 \mathrm{~dB}$. This means that Source 1 is attenuated by $80 \mathrm{~dB}$ and Source 2 only experiences a $1 \mathrm{~dB}$ insertion loss. As a result, the approximate input power at the LNA due to Source 1 and Source 2 is, respectively, $-9 \mathrm{dBm}$ and $39 \mathrm{dBm}$. The front end filter is successful in attenuating the OoB interference from Source 1 below the damage levels. However, the in-band interference of Source 2 exceeds the threshold by $5 \mathrm{~dB}$. The results are summarized in Table II. It is important to understand that we purposely chose Source 1 to be OoB. If it was in-band, the received power would exceed the damage level of the LNA by $35 \mathrm{~dB}$, so we can conclude that the front end filter is very effective in mitigating the impact of $\mathrm{OoB}$ interference.

\section{Identification of PRotection Technologies}

There are few methods available for the protection of frontdoor coupling since the additional measures taken to attenuate the incoming interferences will also contribute to degrading the operation of the system in normal conditions. Therefore, the chosen methods need to provide a very low insertion loss.

\section{A. Fencing}

A possible way to diminish the amplitude of the incoming disturbance is to restrict the minimum distance to which a source could approach the communication tower. The minimum distance can be restricted with the use of a protection fence at a given radius from the tower, so as to increase the

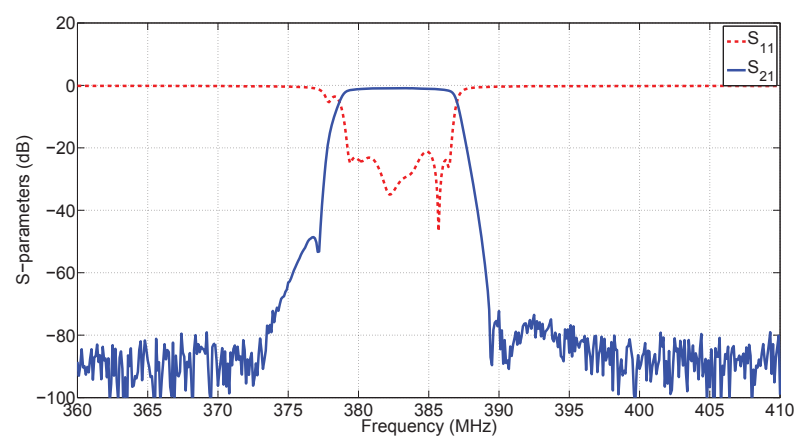

Fig. 4. S-parameters of a BPF as a function of frequency. Adopted from [6].

TABLE II. REQUIRED PROTECTION LEVELS.

\begin{tabular}{c|c|c|c|c}
\hline $\begin{array}{c}\text { Source } \\
\text { ID }\end{array}$ & $\begin{array}{c}\text { Incident field } \\
\text { strength } E \\
(\mathbf{k V / m})\end{array}$ & $\begin{array}{c}\text { Filter } \\
\text { attenuation } \\
(\mathbf{d B})\end{array}$ & $\begin{array}{c}\text { Power at the } \\
\text { LNA }(\mathbf{d B m})\end{array}$ & $\begin{array}{c}\text { Required } \\
\text { Protection } \\
(\mathbf{d B})\end{array}$ \\
\hline \hline $\begin{array}{c}\text { Source } \\
1\end{array}$ & 11.25 & 80 & -9 & N/A \\
\hline $\begin{array}{c}\text { Source } \\
2\end{array}$ & 0.33 & 1 & 39 & 5 \\
\hline
\end{tabular}

separation distance $S$. The additional attenuation compared to $r=20 \mathrm{~m}$ as a function of the radius can be estimated with the following formula:

$$
A t t=20 \log _{10}\left(\frac{\sqrt{S^{2}+H^{2}}}{20}\right)
$$

where $S$ is the separation and $H$ the height of the base station as indicated in Fig. 2. The additional attenuation by installing a fence at a $S$ of $35 \mathrm{~m}$ is $5.2 \mathrm{~dB}$. However, if we would like to add a $10 \mathrm{~dB}$ safety margin, the fence would have to be at a distance of $112 \mathrm{~m}$. In the previous derivation, we even neglected the fact that with increasing $S$, the IEMI gets closer to the boresight of receiving dipole, i.e. the gain of the dipole antenna increases (see (2)). If this is taken into account, the distance of the fence should be further increased. However, base station towers are often situated at populated areas and therefore it is not easy to put a fence with a large radius around a base station. This all shows that a fence is not a practical solution to protect the base station and is not further taken into account as a protection strategy.

\section{B. RF Limiter}

Perhaps the only effective (commercially available) measure against front-door attacks of LNAs is the use of RF limiters based on PIN diodes. Several vendors provide discrete pin diodes that can be used to limit the incoming power to an LNA. Typical limitation levels are about $20 \mathrm{dBm}$ for input powers between $1 \mathrm{~W}-1000 \mathrm{~W}$. The insertion loss of these devices can be typically between 0 to $3.5 \mathrm{~dB}$ which is a very good rating for the front-door protection.

For the application, the required pin diodes usually work in the band between $50 \mathrm{MHz}$ and $4 \mathrm{GHz}$. Since the considered sources are pulsed sources, the $\mathrm{CW}$ power that the pin diodes should tolerate is low. Notice that the installation of an RF limiter will provide protection to the LNA from damage but will impede the operation of the system during an attack since communication is not possible if the limiter is active. 
TABLE III. ELEMENTS PARTICIPATING IN THE COST OF IMPLEMENTING A PROTECTION METHOD.
TABLE IV. ESTIMATION OF THE COSTS OF IMPLEMENTING RF LIMITER PROTECTION SCHEME.

\begin{tabular}{ll}
\hline Cost & Description \\
\hline \hline$C_{\mathrm{INIT}}$ & Initial cost \\
$P$ & $\begin{array}{l}\text { Price if protection elements are available for purchase (price per } \\
\text { element times the number of protection elements). }\end{array}$ \\
$H_{\mathrm{HW}}$ & Hours of HW design $(\geq 0)$. \\
$P P H_{\mathrm{HW}}$ & Price-per-hour of HW design. \\
$H_{\mathrm{SW}}$ & Hours of SW design $(\geq 0)$. \\
$P P H_{\mathrm{SW}}$ & Price-per-hour of SW design. \\
$C_{\mathrm{PU}}$ & Cost of material per unit. One unit can be a surface unit, length unit, \\
$U$ & an element, etc. \\
$H_{\mathrm{IN}}$ & Number of units $(\geq 0)$. \\
$P P H_{\mathrm{IN}}$ & Hours of installation. \\
$C_{\mathrm{YOP}}$ & Price-per-hour of installation. \\
$C_{\mathrm{YM}}$ & Yearly operational cost (handling, power consumption, etc.). \\
$H_{\mathrm{HW}, \mathrm{YM}}$ & Yearly maintenance cost. \\
$P P H_{H W} M$ & Hours of HW maintenance $(\geq 0)$. \\
$H_{\mathrm{SW}, \mathrm{YM}}$ & Price-per-hour of HW maintenance. \\
$P P H_{\mathrm{SW}, \mathrm{M}}$ & Hours of SW maintenance $(\geq 0)$. \\
$C_{\mathrm{YT}}$ & Price-per-hour of SW maintenance. \\
& Cost per yearly testing. \\
\hline
\end{tabular}

\section{Vi. Evaluation of the Protection Technology}

\section{A. Monetary Cost}

To assess the monetary cost of implementing a protection scheme, we divide the total life-cycle expenditures into three main categories: initial cost, yearly operational cost, and yearly maintenance cost. The initial cost greatly depends on whether the required equipment and components are available for purchase or if they will need to be designed and manufactured. It also depends on the effort required for the installation of the protection scheme. A yearly operational cost exists if a protection scheme requires the presence of an operator to run it, or if it necessitates an energy supply or any other resources needed for its uninterrupted operation. A yearly maintenance cost is determined by the hardware and/or software maintenance costs, and the cost of yearly checks, if applicable. We propose here the following formulas for calculating the mentioned categories of the protection cost:

$$
\begin{aligned}
C_{\mathrm{INIT}}= & P+\left\{H_{\mathrm{HW}} \cdot P P H_{\mathrm{HW}}+H_{\mathrm{SW}} \cdot P P H_{\mathrm{SW}}\right. \\
& \left.+C_{\mathrm{PU}} \cdot U\right\}+H_{\mathrm{IN}} \cdot P P H_{\mathrm{IN}} \\
C_{\mathrm{YM}}= & H_{\mathrm{HW}, \mathrm{YM}} \cdot P P H_{\mathrm{HW}}+H_{\mathrm{SW}, \mathrm{YM}} \cdot P P H_{\mathrm{SW}} \\
& +C_{\mathrm{YT}}
\end{aligned}
$$

The cumulative cost after $N_{\mathrm{Y}}$ years of use is given by:

$$
C=C_{\mathrm{INIT}}+\left(C_{\mathrm{YOP}}+C_{\mathrm{YM}}\right) \cdot N_{\mathrm{Y}}
$$

The variables in Eq. (4) to (6) are defined in Table III.

The costs of installing an RF limiter equals the initial costs, as there are no yearly operational or yearly maintenance costs. The hourly rates to be used in (4) depend not only on the type of work but also on the particular country where the work is carried out. For simplicity, and since it is not our goal here to calculate the required budget exactly, we will assume the following rates in Euros: $P P H_{\mathrm{HW}}=100$ and $P P H_{\mathrm{IN}}=50$. An approximate estimate of the costs of installing a limiter is presented in Table IV. The costs presented in Table IV are an educated guess based on experience and discussions with

\begin{tabular}{l|c|c|c|c|c|c}
\hline Description & $\begin{array}{c}P \\
(€)\end{array}$ & $H_{\mathrm{HW}}$ & $\begin{array}{c}C_{\mathrm{PU}} \\
(€)\end{array}$ & $U$ & $H_{\mathrm{IN}}$ & $\begin{array}{c}C_{\mathrm{INIT}} \\
(€)\end{array}$ \\
\hline \hline $\begin{array}{l}\text { RF limiter max. CW } \\
\text { power 1000W }\end{array}$ & 2000 & 20 & 500 & 1 & 20 & 5500 \\
\hline $\begin{array}{l}\text { RF limiter max. CW } \\
\text { power 100W }\end{array}$ & 200 & 20 & 500 & 1 & 20 & 3700 \\
\hline $\begin{array}{l}\text { RF limiter max. CW } \\
\text { power 10W }\end{array}$ & 100 & 20 & 500 & 1 & 20 & 3600 \\
\hline
\end{tabular}

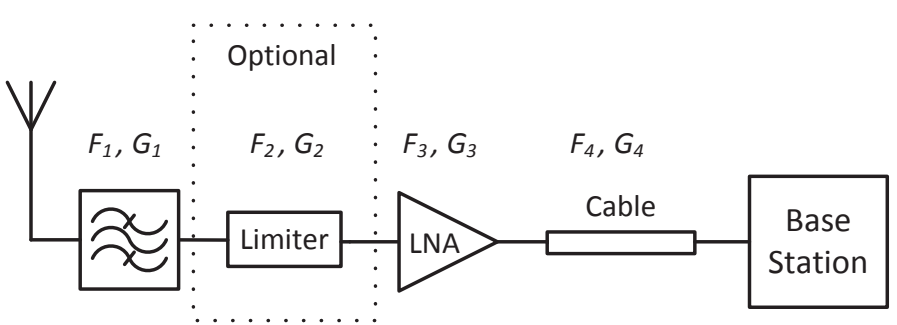

Fig. 5. Receiver configuration

TABLE V. TYPICAL VALUES FOR THE GAIN AND THE NOISE FIGURE OF RECEIVER COMPONENTS.

\begin{tabular}{l|c|c}
\hline Component & Noise Figure $(\mathbf{d B})$ & Gain $(\mathbf{d B})$ \\
\hline \hline 1. Filter & 1 & -1 \\
\hline 2. Limiter & 1 & -1 \\
\hline 3. LNA & 2 & 20 \\
\hline 4. Cable & 4 & -4 \\
\hline
\end{tabular}

experts in this field. The price of adding an RF limiter to a base station is primarily influenced by the costs of material and personnel. However, these costs are fixed, whereas the price of the RF limiter itself depends on the power it should withstand: the higher the power, the higher the price.

\section{B. Loss in Performance}

Another cost of using an RF limiter as a protection scheme is the loss in performance. Let us investigate the receiver noise factor $F$ (or noise figure when expressed in decibels) in the presence of an RF limiter. Fig. 5 shows a typical receiver configuration. The noise factor $\mathrm{F}$ equals

$$
F=\frac{S N R_{\text {in }}}{S N R_{\text {out }}}
$$

where $S N R_{\text {in }}$ and $S N R_{\text {out }}$ are the input and output signalto-noise ratios (SNRs) of the receiver, respectively. The total noise factor, $F_{\text {total }}$, depends on the noise factor of individual components and on their respective gains, and can be estimated using the Friis' formula:

$$
F_{\text {total }}=F_{1}+\frac{F_{2}-1}{G_{1}}+\frac{F_{3}-1}{G_{1} G_{2}}+\frac{F_{4}-1}{G_{1} G_{2} G_{3}}
$$

Typical values of the gain and the noise factor are given in Table V. For the passive components, such as the filter, the limiter, and the cable, it is known that the noise factor equals the insertion loss of the component itself [11]. Without the limiter, $F_{\text {total }}$ is $3 \mathrm{~dB}$. Installing a limiter with an insertion loss $L$ of $1 \mathrm{~dB}$ will degrade $F_{\text {total }}$ by $1 \mathrm{~dB}$. This results in 1 $\mathrm{dB}$ loss of sensitivity. From (8), we can see that $F_{\text {total }}$ depends linearly on the noise factor of the limiter, as $F_{2}=L_{2}$ and $G_{2}=1 / L_{2}$. Fig. 6 shows $F_{\text {total }}$ as a function of the limiter 


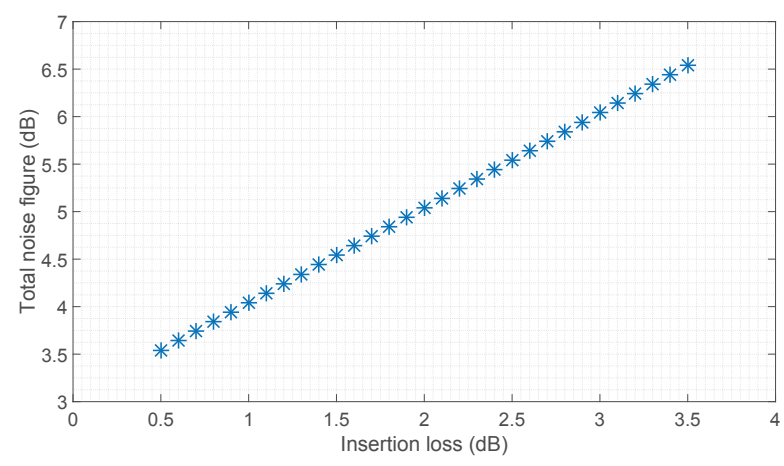

Fig. 6. $F_{\text {total }}$ as a function of limiter insertion loss.

insertion loss $L_{2}$. We varied the insertion loss $L_{2}$ from $0.5 \mathrm{~dB}$ to $3.5 \mathrm{~dB}$ in steps of $0.1 \mathrm{~dB}$. As expected, every additional $\mathrm{dB}$ of insertion loss affects $F_{\text {total }}$. This is one of the main reasons why limiters are not often present in a receivers.

\section{CONCLUSION}

In this paper, we analyzed the susceptibility of a wireless infrastructure and presented a methodology to estimate the required protection levels. The focus in this paper was mainly on a communication infrastructure based on TETRA, but it can be applied to any critical infrastructure. We showed that available sources at a distance of $20 \mathrm{~m}$ can easily generate an input power at the LNA that exceeds the susceptibility level of the LNA. The best protection against OoB interference is a front end filter, which significantly reduces the vulnerability. However, wireless receivers showed to be highly vulnerable against in-band interference.

A possible protection technique is the installation of an RF limiter in the front end of the receiver. The possibility of restricting the minimum distance to which a source could approach the communication tower showed not to be effective. IEMI can effectively be coupled into the antenna at a large distance.

We presented a method to estimate the costs of a protection technique. We applied this method on the installation of an RF limiter in a wireless receiver. It showed that the price of adding an RF limiter to a base station is primarily influenced by the costs of material and personnel. The costs presented here are intended only to illustrate the methodology. The actual costs for any particular implementation will depend greatly on the detailed specification, quantities to be installed and whether the protection is installed as the infrastructure is built, or fitted to an existing installation.

Finally, we showed that the loss in performance of a wireless receiver can be expressed using the noise factor. It is essential to keep the insertion loss of the limiter as low as possible to mitigate the loss in performance of the wireless receiver.

\section{ACKNOWLEDGMENT}

The research leading to these results has received funding from the European Union Seventh Framework Programme under grant agreement number FP7-SEC-2011-285257.

\section{REFERENCES}

[1] W. A. Radasky, C. E. Baum, and M. W. Wik, "Introduction to the special issue on high-power electromagnetics (HPEM) and intentional electromagnetic interference (IEMI)," Electromagnetic Compatibility, IEEE Transactions on, vol. 46, no. 3, pp. 314-321, 2004.

[2] B. Menssen, M. Mleczko, H. Garbe, K.-U. Rathjen, S. Dickmann, S. van de Beek, and F. Leferink, "Reference configurations for the characterization of critical infrastructures," in Electromagnetic Compatibility (EMC Europe), 2014 International Symposium on, Sept 2014, pp. $1218-1223$.

[3] M. Stahlberg, "Radio Jamming Attacks Against Two Popular Mobile Networks," in Helsinki University of Technology Seminar on Network Security., 2000.

[4] M. Backstrom and K. Lovstrand, "Susceptibility of electronic systems to high-power microwaves: summary of test experience," Electromagnetic Compatibility, IEEE Transactions on, vol. 46, no. 3, pp. 396-403, Aug 2004.

[5] D. Mansson, R. Thottappillil, M. Backstrom, and O. Lunden, "Vulnerability of European Rail Traffic Management System to Radiated Intentional EMI," Electromagnetic Compatibility, IEEE Transactions on, vol. 50, no. 1, pp. 101-109, 2008.

[6] S. van de Beek and F. Leferink, "Robustness of a TETRA Base Station Receiver Against Intentional EMI," Electromagnetic Compatibility, IEEE Transactions on, vol. 57, no. 3, pp. 461-469, June 2015.

[7] T. Nilsson and R. Jonsson, "Investigation of HPM front-door protection devices and component susceptibility," FOI - Swedish Defence Research Agency, Tech. Rep. FOI-R-1771-SE, 2005.

[8] J. Gavan and M. Shulman, "Effects of densensitization on mobile radio system performance, part I: Qualitative analysis," Vehicular Technology, IEEE Transactions on, vol. 33, no. 4, pp. 285-290, Nov 1984.

[9] K. Pelechrinis, M. Iliofotou, and S. V. Krishnamurthy, "Denial of Service Attacks in Wireless Networks: The Case of Jammers," Communications Surveys \& Tutorials, IEEE, vol. 13, no. 2, pp. 245-257, 2011.

[10] C. Balanis, Antenna Theory, 2nd ed. New York: John Wiley \& Sons, 1997.

[11] W. Van Etten, Introduction to Random Signals and Noise. John Wiley \& Sons, Ltd, 2006.

[12] D. C. Schleher, Electronic Warfare in the Information Age. Artech House, Inc, 1999.

[13] N. Mora, F. Vega, G. Lugrin, F. Rachidi, and M. Rubinstein, "Study and Classification of Potential IEMI Sources," System Design and Assessment Notes, Note 41, 2014.

[14] "DIEHL 2011. HPEM Case Brochure. In: DIEHL (ed.)."

[15] F. Vega, "Analytical methods for the study and design of integrated switched oscillators and antennas for mesoband radiation," Ph.D. dissertation, École Polytechnique Fédérale de Lausanne, 2013.

[16] Terrestrial Trunked Radio (TETRA); Feasibility Study into the Implications of Operating Public Safety Sector (PSS) TEDS using the proposed "Tuning Range" concept in the $410 \mathrm{MHz}$ to $430 \mathrm{MHz}$ and $450 \mathrm{MHz}$ to $470 \mathrm{MHz}$ frequency bands, ETSI TR 102 513, V1.1.1, 2006. 